\title{
Unravelling defect passivation mechanisms in sulfur-treated $\mathrm{Sb}_{2} \mathrm{Se}_{3}$
}

Rajiv Ramanujam Prabhakar, ${ }^{1}$ Thomas Moehl, ${ }^{1}$ Dennis Friedrich, ${ }^{2}$ Marinus Kunst, ${ }^{2}$ Sudhanshu Shukla, ${ }^{3}$ Damilola Adeleye, ${ }^{3}$ Vinayaka H. Damle, ${ }^{4}$ Sebastian Siol, ${ }^{5}$ Wei Cui, ${ }^{1}$ Laxman Gouda, ${ }^{1}$ Jihye Suh, ${ }^{1}$ Yaakov R. Tischler ${ }^{4}$, Roel van de Krol, ${ }^{2}$ and S. David Tilley ${ }^{1 *}$

${ }^{1}$ Department of Chemistry, University of Zurich, Switzerland

${ }^{2}$ Helmholtz-Zentrum Berlin für Materialien und Energie GmbH (HZB), Germany

${ }^{3}$ University of Luxembourg, Luxembourg

${ }^{4}$ Bar-Ilan University, Israel

${ }^{5}$ EMPA, Switzerland

Keywords: $\mathrm{Sb}_{2} \mathrm{Se}_{3}$, charge carrier dynamics, time-resolved microwave conductivity, photoluminescence spectroscopy, low-frequency Raman spectroscopy 


\begin{abstract}
$\mathrm{Sb}_{2} \mathrm{Se}_{3}$ has emerged as an important photoelectrochemical (PEC) and photovoltaic (PV) material due to its rapid rise in photoconversion efficiencies. However, despite its binary nature, $\mathrm{Sb}_{2} \mathrm{Se}_{3}$ has a complex defect chemistry, which reduces the maximum photovoltage that can be obtained. Thus, it is important to understand these defects and to develop passivation strategies in order to further improve this material. In this work, a comprehensive investigation of the charge carrier dynamics of $\mathrm{Sb}_{2} \mathrm{Se}_{3}$ and the influence of sulfur treatment on its optoelectronic properties was performed using time resolved microwave conductivity (TRMC), photoluminescence (PL) spectroscopy and low frequency Raman spectroscopy (LFRS). The key finding in this work is that upon sulfur treatment of $\mathrm{Sb}_{2} \mathrm{Se}_{3}$, the carrier lifetime is increased by the passivation of deep defects in $\mathrm{Sb}_{2} \mathrm{Se}_{3}$ in both the surface region and the bulk, which is evidenced by increased charge carrier lifetime of TRMC decay dynamics, increased radiative recombination efficiency and decreased deep defect level emission (PL), and improved long-range order in the material (LFRS). These findings provide crucial insights into the defect passivation mechanisms in $\mathrm{Sb}_{2} \mathrm{Se}_{3}$ paving the way for developing highly efficient PEC and PV devices.
\end{abstract}




\section{INTRODUCTION}

Photovoltaic (PV) and photoelectrochemical (PEC) cells based on $\mathrm{Sb}_{2} \mathrm{Se}_{3}$ have seen a rapid rise in interest in recent years and significant progress has been made in their power conversion efficiencies, ${ }^{[1,2]}$ which is primarily due to its high absorption coefficient and excellent optoelectronic properties. However, a major challenge for $\mathrm{Sb}_{2} \mathrm{Se}_{3}$ is the low photovoltage obtained from such devices. Originally it was thought that $\mathrm{Sb}_{2} \mathrm{Se}_{3}$ would exhibit low defect concentration as it is a binary compound (better stoichiometric control) with benign grain boundaries. ${ }^{[3]}$ However, recent computational studies have shown the complex defect chemistry of $\mathrm{Sb}_{2} \mathrm{Se}_{3}$ and the formation of deep defect states that are detrimental to PV/PEC performance. ${ }^{[4]}$ Cation-anion anti-site defects have been shown to have a low formation energy and are energetically positioned close to the mid gap, serving as deep recombination centers. Therefore, the understanding and the suppression/passivation of such deep defects is crucial for the further development of $\mathrm{Sb}_{2} \mathrm{Se}_{3}$ as a $\mathrm{PV} / \mathrm{PEC}$ material. Several strategies have been presented in the literature such as Te incorporation for defect passivation, ${ }^{[5]}$ sulfurization treatment for improved photovoltage ${ }^{[6]}$ and gradient sulfur doping in $\mathrm{Sb}_{2} \mathrm{Se}_{3} .{ }^{[7]}$ More fundamental insights into the basic optoelectronic properties for defect passivation/suppression for $\mathrm{Sb}_{2} \mathrm{Se}_{3}$, however, have not yet been reported.

In this work, we employed time resolved microwave conductivity (TRMC) and photoluminescence (PL) spectroscopy to gain fundamental understanding of the defect passivation mechanism in $\mathrm{Sb}_{2} \mathrm{Se}_{3}$ by sulfur treatment. We investigated the charge carrier dynamics of $\mathrm{Sb}_{2} \mathrm{Se}_{3}$ before and after sulfur treatment using TRMC and observed the passivation of deep defects in $\mathrm{Sb}_{2} \mathrm{Se}_{3}$ as evidenced by longer carrier lifetimes. We also show that the passivation of deep defects reduces not only surface recombination, but also bulk recombination using a wavelength dependent TRMC study. Furthermore, we demonstrate increased radiative recombination efficiency by suppression of nonradiative recombination pathways in sulfur-treated $\mathrm{Sb}_{2} \mathrm{Se}_{3}$ using PL spectroscopy. In addition to this, low frequency Raman spectroscopy (LFRS) showed increased long-range order in the $\mathrm{Sb}_{2} \mathrm{Se}_{3}$ semiconductor thin films upon sulfur treatment. These results explain the improved photovoltage of $\mathrm{Sb}_{2} \mathrm{Se}_{3}$ upon sulfur treatment, which presents a simple and efficient defect passivation strategy. 


\section{RESULTS AND DISCUSSION}

$\mathrm{Sb}_{2} \mathrm{Se}_{3}$ thin films were synthesized on quartz substrates by selenization of $\mathrm{Sb}$ metal. The sulfurization of these films was carried out in a sulfur atmosphere (see experimental section for details). No significant surface morphological changes of $\mathrm{Sb}_{2} \mathrm{Se}_{3}$ thin films were observed upon sulfur treatment and incorporation of sulfur into the films was confirmed by EDX (Figure S1). The XRD pattern of $\mathrm{Sb}_{2} \mathrm{Se}_{3}$ was indexed to the orthorhombic phase of $\mathrm{Sb}_{2} \mathrm{Se}_{3}$ (JCPDS 01-0890821), and that of the sulfur-treated film was identical to the $\mathrm{Sb}_{2} \mathrm{Se}_{3}$ (Figure S2), indicating that the bare $\mathrm{Sb}_{2} \mathrm{Se}_{3}$ crystal structure was not affected by the sulfurization treatment. It is also evident from the absorption spectrum, that the onset of absorption does not change significantly with sulfur treatment indicating that the incorporation of sulfur does not change the optical band gap (Figure S3).

We investigated the charge carrier dynamics in $\mathrm{Sb}_{2} \mathrm{Se}_{3}$ and how sulfur treatment of $\mathrm{Sb}_{2} \mathrm{Se}_{3}$ affects the underlying carrier recombination processes using TRMC. In TRMC, photoexcited charge carriers are generated in a semiconductor by a short laser pulse, giving rise to a light-induced change of the conductance in the sample (photoconductance $(\Delta \mathrm{G})$, Figure 1 a) as compared to the dark conductance. ${ }^{[8,9]}$ This photoconductance changes over time due to both radiative and nonradiative losses of free mobile charge carriers in the semiconductor and hence valuable information with regards to the loss mechanism of photogenerated charge carriers in the semiconductor can be obtained. The bare $\mathrm{Sb}_{2} \mathrm{Se}_{3}$ and sulfur-treated films were excited with $\sim 3$ ns pulses (with wavelengths between $350-1100 \mathrm{~nm})$ at different intensities $\left(10^{9}-10^{13}\right.$ photons $\left./ \mathrm{pulse} / \mathrm{cm}^{2}\right)$ and the change in photoconductance was monitored over time (ns to $\sim 80 \mu \mathrm{s}$ ). As there are no charge selective contacts to separate the charge carriers, and assuming that the carrier mobility does not change during the time window of our measurement, the decrease in the photoconductance with time is purely due to the decrease in the concentration of mobile carriers in the bands by trapping or annihilation by recombination. Figure $\mathbf{S 4}$ shows the photoconductance normalized to the amount of photogenerated charge carriers $\left(\Delta \mathrm{G} / \beta \mathrm{eI} \mathrm{I}_{0} \mathrm{~F}_{\mathrm{A}}\right)$ as a function of time under $500 \mathrm{~nm}(2.48$ $\mathrm{eV}$ ) pulsed illumination with a light intensity of $3.69 \times 10^{9}$ photons $/ \mathrm{cm}^{2}$ per pulse (light intensity corresponding to 1 sun illumination where the laser power was chosen such that the number of photons absorbed would equal to what it would be under 1 sun) for $\mathrm{Sb}_{2} \mathrm{Se}_{3}$ and sulfur-treated films. From the peak of the TRMC signal (the maximum photoconductance of the sample), we can estimate the sum of the mobilities of the individual carriers using the equation given below ${ }^{[10]}$ 


$$
\Phi \Sigma \mu=\frac{\Delta G_{\max }}{I_{0} \beta e F_{A}}
$$

where $I_{0}$ is the incident intensity per pulse, $\mathrm{e}$ is the elementary charge, $\beta$ is the ratio between the inner broad and narrow dimensions of the waveguide, $\mathrm{F}_{\mathrm{A}}$ is the fraction of incident photons absorbed within the sample, $\varphi$ is the charge carrier generation yield, and $\Sigma \mu$ is the sum of electron and hole mobilities (Figure 1 b). The effective mobility for both $\mathrm{Sb}_{2} \mathrm{Se}_{3}$ and sulfur-treated films was greater than $10 \mathrm{~cm}^{2} \mathrm{~V}^{-1} \mathrm{~s}^{-1}$, which is considerably higher than other water splitting materials such as $\mathrm{Fe}_{2} \mathrm{O}_{3}\left(0.005 \mathrm{~cm}^{2} \mathrm{~V}^{-1} \mathrm{~s}^{-1}\right),{ }^{[11]} \mathrm{CuFeO}_{2}\left(0.2 \mathrm{~cm}^{2} \mathrm{~V}^{-1} \mathrm{~s}^{-1}\right),{ }^{[12]}$ and $\mathrm{BiVO}_{4}\left(0.08 \mathrm{~cm}^{2} \mathrm{~V}^{-1} \mathrm{~s}^{-1}\right)^{[13]}$. Interesting to note is the fact that at a relatively low intensity of photons from the laser $\left(\sim 10^{9}\right.$ photons $/ \mathrm{cm}^{2}$ per pulse) clear TRMC signals could be detected in contrast to the light intensities of $>10^{13}$ photons $/ \mathrm{cm}^{2} /$ pulse that are needed to detect signals from many other absorber materials. This observation reveals the excellent optoelectronic properties and low trap density of $\mathrm{Sb}_{2} \mathrm{Se}_{3}{ }^{[14]}$ The maximum of the (non-normalized) peak height is quite similar for the two different sample types showing that the sulfur treatment did not substantially change the effective mobility values of the charge carriers. 

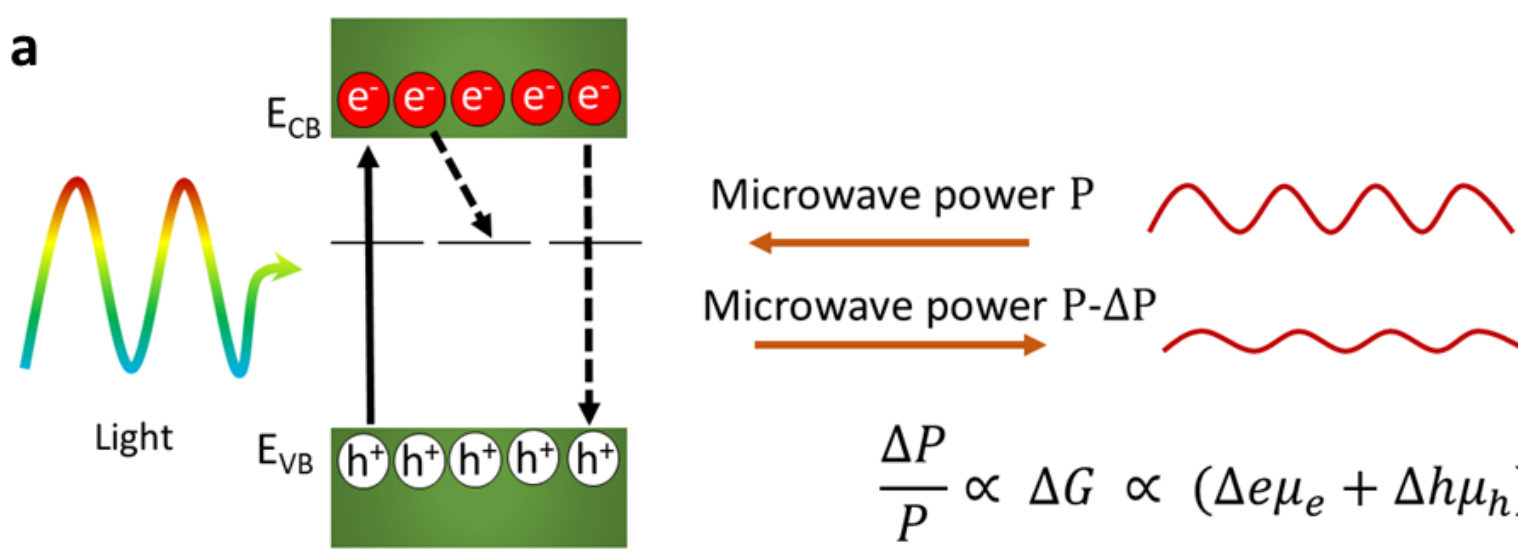

Microwave power P- $\Delta \mathrm{P}$

$$
\frac{\Delta P}{P} \propto \Delta G \propto\left(\Delta e \mu_{e}+\Delta h \mu_{h}\right)
$$
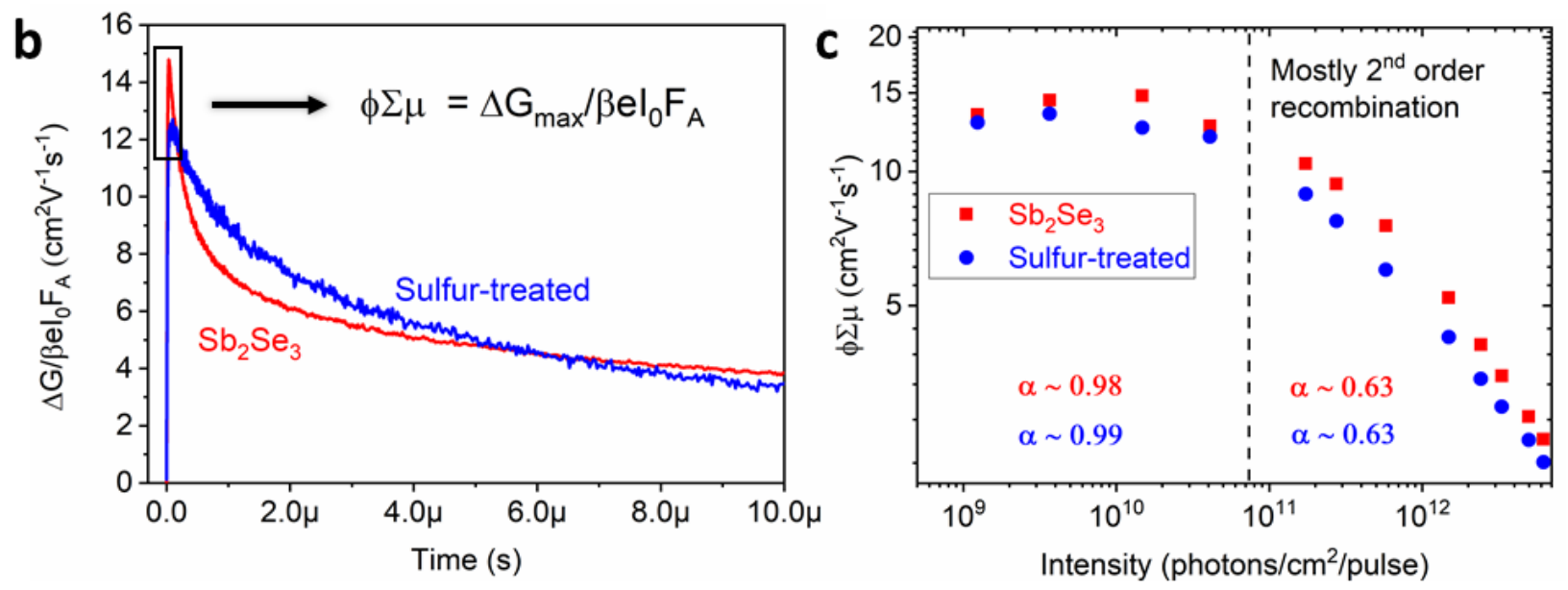

Figure 1: Photoconductance and recombination kinetics of $\mathrm{Sb}_{2} \mathrm{Se}_{3}$ and sulfur-treated films.

a Schematic illustrating the principle of TRMC. Change in the microwave power $\triangle \mathrm{P}$ is proportional to the change in photoconductance $\Delta \mathrm{G}$, which is related to the light induced concentration of photogenerated electrons $(\Delta \mathrm{n})$ and holes $(\Delta \mathrm{p})$ and the electron mobility $\mu_{\mathrm{e}}$ and hole mobility $\mu_{\mathrm{h}}$. b Photoconductance vs time plots for $\mathrm{Sb}_{2} \mathrm{Se}_{3}$ and sulfur-treated films (estimation of $\Phi \Sigma \mu$ from the peak photoconductance at a light intensity of $1.48 \times 10^{10}$ photons $/ \mathrm{cm}^{2} / \mathrm{pulse}$ ) under $500 \mathrm{~nm}$ illumination. c $\Phi \Sigma \mu$ vs light intensity plots for $\mathrm{Sb}_{2} \mathrm{Se}_{3}$ and sulfur-treated films showing higher order recombination regimes, where $\alpha$ is the reciprocal of the order of the recombination process.

The charge carriers generated are proportional to the incident light intensity on the semiconductor, and the recombination of charge carriers can be understood using the general recombination rate equation given by ${ }^{[15]}$ 


$$
\frac{d n}{d t}=-a n-b n^{2}-c n^{3}
$$

where $\mathrm{n}$ is the photocarrier density, and $\mathrm{a}, \mathrm{b}$, and $\mathrm{c}$ are recombination rate constants. If the change in photocarrier density $\mathrm{dn} / \mathrm{dt}$ (controlled by a change in incident light intensity) shows a linear dependence, then first order recombination is the dominant recombination process (i.e., $\mathrm{a} \approx 1$ and b,c $<<1$ ), while a sub-linear dependence would indicate higher order recombination processes.

Upon comparing the light intensity dependence of the maximum of the photoconductance signal, it is observed that the TRMC signal is relatively constant at lower light intensities and then decreases with increasing light intensity $>10^{11}$ photons $/ \mathrm{cm}^{2} /$ pulse (Figure $1 \mathrm{c}$ ). Based on the slope of the log plot $\Phi \Sigma \mu$ vs $\mathrm{I}_{0}{ }^{\alpha-1}$, the order of the mobile charge carrier loss process could be deduced (where $\alpha$ is the reciprocal of the order of the recombination process). ${ }^{[13]}$ At lower light intensities, the concentration of photogenerated electrons and holes is relatively low and trapping is the dominant loss process for mobile charge carriers. At light intensities above $10^{11}$ photons $/ \mathrm{cm}^{2} / \mathrm{pulse}$ $\alpha$ was $\sim 0.63$, showing that second order recombination dominates. At these increased light intensities, the bands are populated by a high density of photogenerated charge carriers leading to increased second order recombination-probably due to direct electron and hole recombination-and a reduction in the TRMC signal height (Figure 1 c) already during the laser pulse. Moreover, it is interesting to note that the onset for second order recombination occured at lower photon flux in sulfur-treated films (as indicated by extrapolation in Figure S5). The explanation for this phenomenon is that the sulfur treatment reduces the amount of defect/trap states, which enables the accumulation of a higher concentration of photogenerated charge carriers in the bands at lower laser intensities as compared to the untreated sample. Therefore, the second order recombination process sets in at a lower light intensity. 

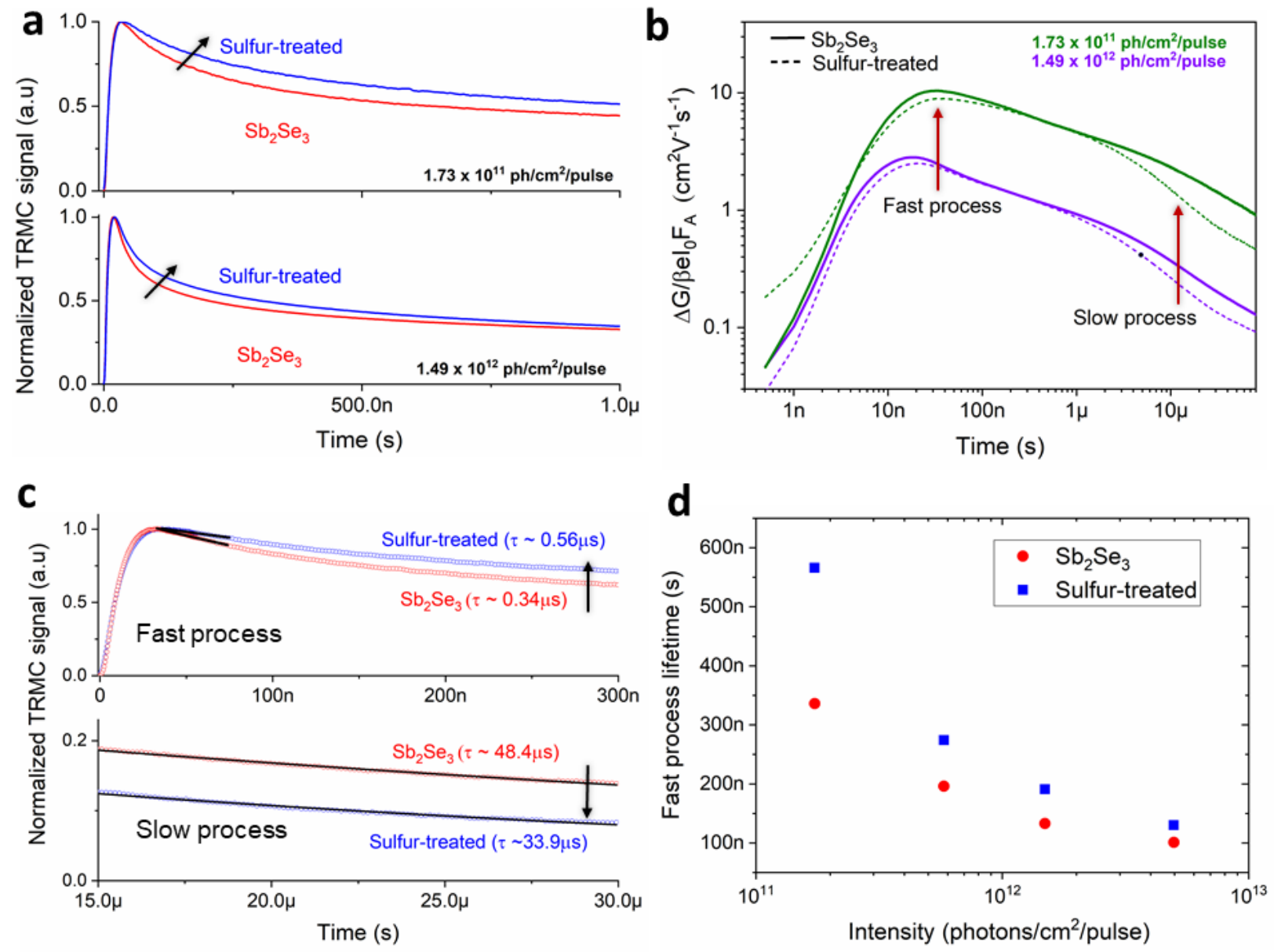

Figure 2. Lifetime of photoconductance decay processes in $\mathrm{Sb}_{2} \mathrm{Se}_{3}$ and sulfur-treated films.

a Normalized TRMC signal showing the longer lifetime of sulfur-treated films under $500 \mathrm{~nm}$ illumination. b Double log plot for $\mathrm{Sb}_{2} \mathrm{Se}_{3}$ and sulfur-treated films showing the different decay processes taking place under $500 \mathrm{~nm}$ illumination. $\mathbf{c}$ Exponential fitting to estimate the lifetime of the fast and slow processes under $500 \mathrm{~nm}$ illumination $\left(1.73 \times 10^{11}\right.$ photons $\left./ \mathrm{cm}^{2} / \mathrm{pulse}\right)$. d Dependence of the fast process lifetime on light intensity for the $\mathrm{Sb}_{2} \mathrm{Se}_{3}$ and sulfur-treated films.

Qualitative examination of the normalized photoconductance decay at a single wavelength (500 $\mathrm{nm}$ ) for the $\mathrm{Sb}_{2} \mathrm{Se}_{3}$ and the sulfur-treated films (Figure 2 a) shows a slower initial decay for the sulfur-treated film. This observation indicates a decreased loss of mobile charge carriers, which points to a lower amount of defects/traps in the sulfur-treated sample.

Usually, the TRMC decays are either fitted with a single or a double exponential function. However, as is seen from Figure S6, both single and double exponential functions do not provide 
a satisfactory fit with our TRMC decay. In order to understand this better, we made double log plots of TRMC decays (Figure 2 b). It is evident from the plots that there are different decay processes that begin at different times (a fast process occuring in less than $100 \mathrm{~ns}$, and a slow process occuring beyond $15 \mu \mathrm{s}$ ). If the two processes began at the same time, then a double exponential function would fit well with our data. As this is clearly not the case, we treated these processes separately and fitted them each with a single exponential function, as shown in Figure 2 c. This method leads to better fits of our TRMC decay, and the time constants of these processes ( $\tau_{\text {fast }}$ and $\tau_{\text {slow }}$ ) could be determined (Figure $2 \mathbf{c}$ ). The initial fast decay is probably due to defectmediated recombination, which is also the decay process active under moderate stationary illumination, i.e. in PV and PEC devices under standard operational conditions. The difference in $\tau_{\text {fast }}$ between the $\mathrm{Sb}_{2} \mathrm{Se}_{3}$ and the sulfur-treated $\mathrm{Sb}_{2} \mathrm{Se}_{3}$ was found to decrease with increasing light intensity due to progressively dominant higher order recombination (Figure 2d). At the highest light intensities $\left(>10^{12}\right.$ photons $/ \mathrm{cm}^{2} /$ pulse), the difference in lifetime between $\mathrm{Sb}_{2} \mathrm{Se}_{3}$ and sulfurtreated films was found to be minimal as a result of direct electron and hole recombination. The dependence of the decay time of this process on the excitation density (Figure 2d) is difficult to explain, although a defect-mediated recombination process can depend on the excitation density. Another possibility is that direct recombination within the pulse is still active after the pulse, but our fitting with a time-independent residue does not support this interpretation. In any case, an intricate numerical model seems necessary to fully resolve this question. It is noteworthy that upon sulfur treatment, the decay of the fast process is decelerated, indicating a slower loss of mobile charge carriers. The reason can be that the sulfur treatment reduces trap states or recombination. Due to a reduced number of defects following sulfur treatment, the probability of defect mediated mobile charge carrier loss is reduced, hence a longer lifetime after sulfur treatment is observed.

On the other hand, the slow process with its characteristic time constant $\tau_{\text {slow }}$ is shorter for the sulfurized film. This slow process ( $>15 \mu$ s) is likely due to de-trapping of carriers from shallow defect states back into the bands followed by band to band recombination. ${ }^{[16]}$ With reduced and/or shallower defect/trap states, the trapping and de-trapping process is faster for the sulfur-treated samples, leading to a faster repopulation of the bands by thermalization from the traps and increasing the probability of band to band recombination. 

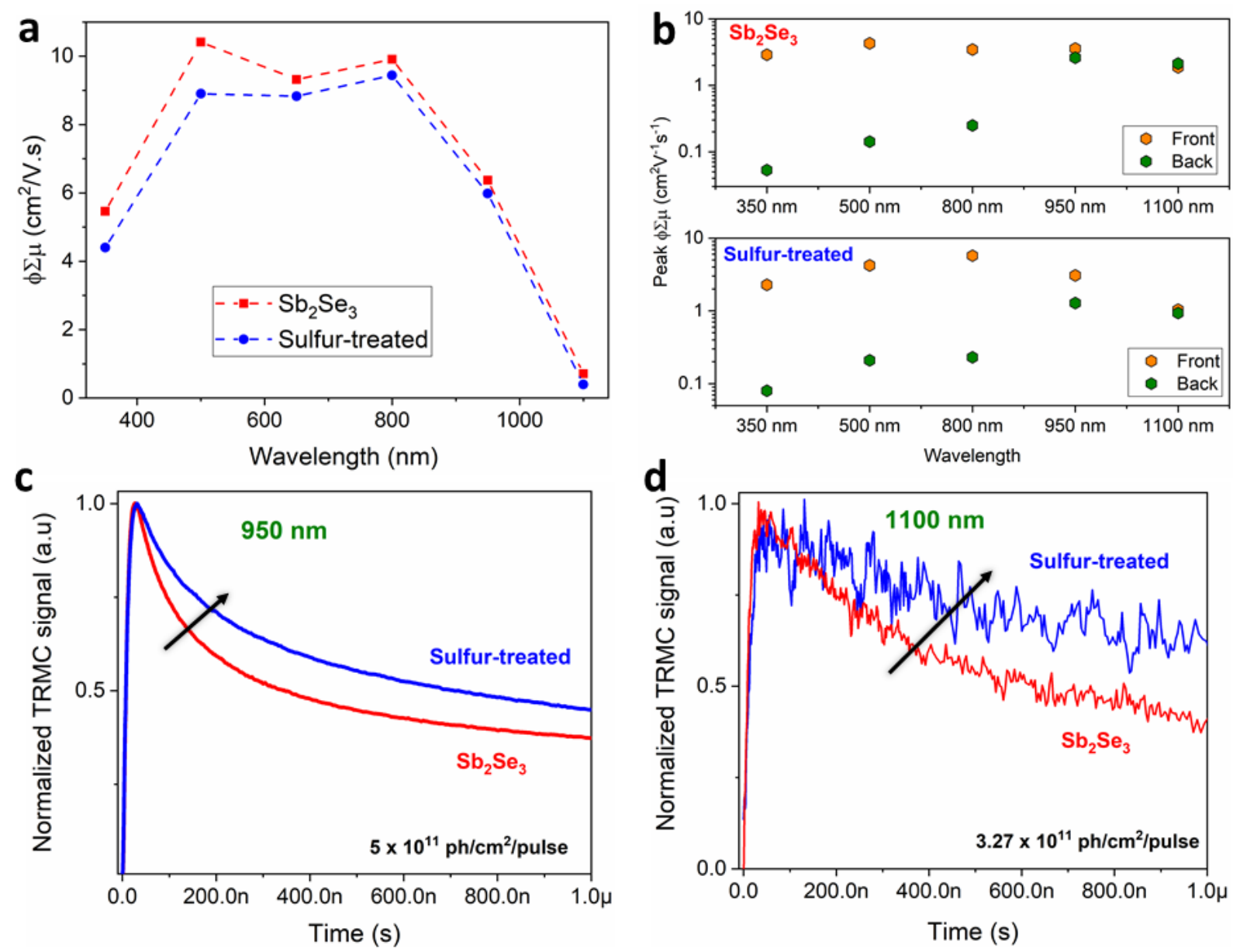

Figure 3. Wavelength dependent TRMC decay dynamics. a $\Phi \Sigma \mu$ vs wavelength plots for $\mathrm{Sb}_{2} \mathrm{Se}_{3}$ and sulfur-treated films (light intensity $\sim 2 \times 10^{11}$ photons $/ \mathrm{cm}^{2} / \mathrm{pulse}$ ). b $\Phi \Sigma \mu$ peak value vs wavelength plots for $\mathrm{Sb}_{2} \mathrm{Se}_{3}$ and sulfur-treated films for front vs back illumination (light intensity $\sim 2 \times 10^{12}$ photons $/ \mathrm{cm}^{2} /$ pulse). Normalized TRMC signal plots for $\mathbf{c} 950 \mathrm{~nm}$ and $\mathbf{d} 1100 \mathrm{~nm}$ illumination of $\mathrm{Sb}_{2} \mathrm{Se}_{3}$ and sulfur-treated films.

Now we consider the wavelength dependence of the TRMC decay. The variation of the $\Phi \Sigma \mu$ signal mirrors the absorption spectrum of $\mathrm{Sb}_{2} \mathrm{Se}_{3}$ and sulfur-treated films except in the case of $350 \mathrm{~nm}$, which shows a sharp decrease in the response (Figure 3 a). The photogenerated charge carriers from $350 \mathrm{~nm}$ illumination would be generated close to the surface due to the high absoprtion coefficient at this photon energy, and would yield a low $\Phi \Sigma \mu$ signal due to surface recombination. ${ }^{[17]}$ The $\Phi \Sigma \mu$ was compared for the different wavelengths using front vs back illumination. The shorter wavelengths (350-800 nm) exhibited significantly lower peak $\Phi \Sigma \mu$ signal 
when illuminated from the back, which is probably due to a high recombination velocity at the back interface (quartz/ $\mathrm{Sb}_{2} \mathrm{Se}_{3}$ ) because of a high defect density. With decreasing energy of the excitation wavelength (950 and $1100 \mathrm{~nm}$ ), the penetration depth increases and the photogenerated charge carriers have a similar and even distribution for both front and back illumination, which is evident by the similar peak $\Phi \Sigma \mu$ signal (Figure 3 b). This observation implies that the $950 \mathrm{~nm}$ and $1100 \mathrm{~nm}$ signal originate from regions deeper in the bulk of the semiconductor. Upon comparison of the TRMC decay of $\mathrm{Sb}_{2} \mathrm{Se}_{3}$ and sulfur-treated films for $950 \mathrm{~nm}$ and $1100 \mathrm{~nm}$ illumination, the sulfur-treated samples exhibited a longer lifetime of the fast process than the $\mathrm{Sb}_{2} \mathrm{Se}_{3} \mathrm{sample}$ (Figure 3 c,d and Figure S7), which clearly shows that the sulfur treatment results in a reduction of the electron hole recombination in the bulk region of the semiconductor. Supporting this observation, XPS depth profiling clearly shows the penetration of sulfur deeper into the film (Figure S8). The initially high concentration of sulfur close to the surface decreases until it settles at approximately $\sim 10$ at. $\%$.

Next we used photoluminescence (PL) spectroscopy to understand the radiative recombination and to identify the passivation of non-radiative recombinative pathways of $\mathrm{Sb}_{2} \mathrm{Se}_{3}$ and sulfur-treated films. As the $\mathrm{Sb}_{2} \mathrm{Se}_{3}$ and sulfur-treated films exhibited low PL yield at room temperature, the PL spectra were acquired at $10 \mathrm{~K}$ (Figure 4 a). The PL yield from $\mathrm{Sb}_{2} \mathrm{Se}_{3}$ was an order of magnitude lower than the sulfur-treated samples, indicating higher non-radiative losses in $\mathrm{Sb}_{2} \mathrm{Se}_{3}$ before sulfur treatment (inset Figure 4 a). After sulfur treatment, these non-radiative recombination pathways are reduced, leading to a higher PL intensity. The PL spectra show a bandedge emission and a broad defect emission at lower energy. Investigation on the band-edge PL peak at $1.25 \mathrm{eV}$ indicates that the transition is likely a free to bound state emission at $1.25 \mathrm{eV}$ (as evidenced by no peak energy shift and the $<1$ power law exponent of the PL intensity $v s$ incident power plot (Figure S9)). ${ }^{[18,19]}$ The defect emission band extends over a broad energy range from 0.75 to $1.15 \mathrm{eV}$ for $\mathrm{Sb}_{2} \mathrm{Se}_{3}$. The defect emission in the PL spectra at energies close to $0.8 \mathrm{eV}$ were reduced due to sulfur treatment suggesting a removal of a deep defect/trap state (Figure S10). The increased radiative recombination and decreased defect emission peak in sulfur-treated films coupled with a longer lifetime of the fast process and reduced bulk recombination observed from TRMC strongly suggest a passivation of deep defects in $\mathrm{Sb}_{2} \mathrm{Se}_{3}$. Recent computational studies have shown that $\mathrm{Sb}_{\mathrm{Se}}$ antisite defects would occupy states at $0.4 \mathrm{eV}$ above the valence band of $\mathrm{Sb}_{2} \mathrm{Se}_{3}$ and the suppresion of defect emission at energies close to $0.8 \mathrm{eV}$ could imply the passivation of $\mathrm{Sb}_{\mathrm{Se}}$ antisite defects upon sulfur treatment of $\mathrm{Sb}_{2} \mathrm{Se}_{3}$ films. ${ }^{[20]}$ 

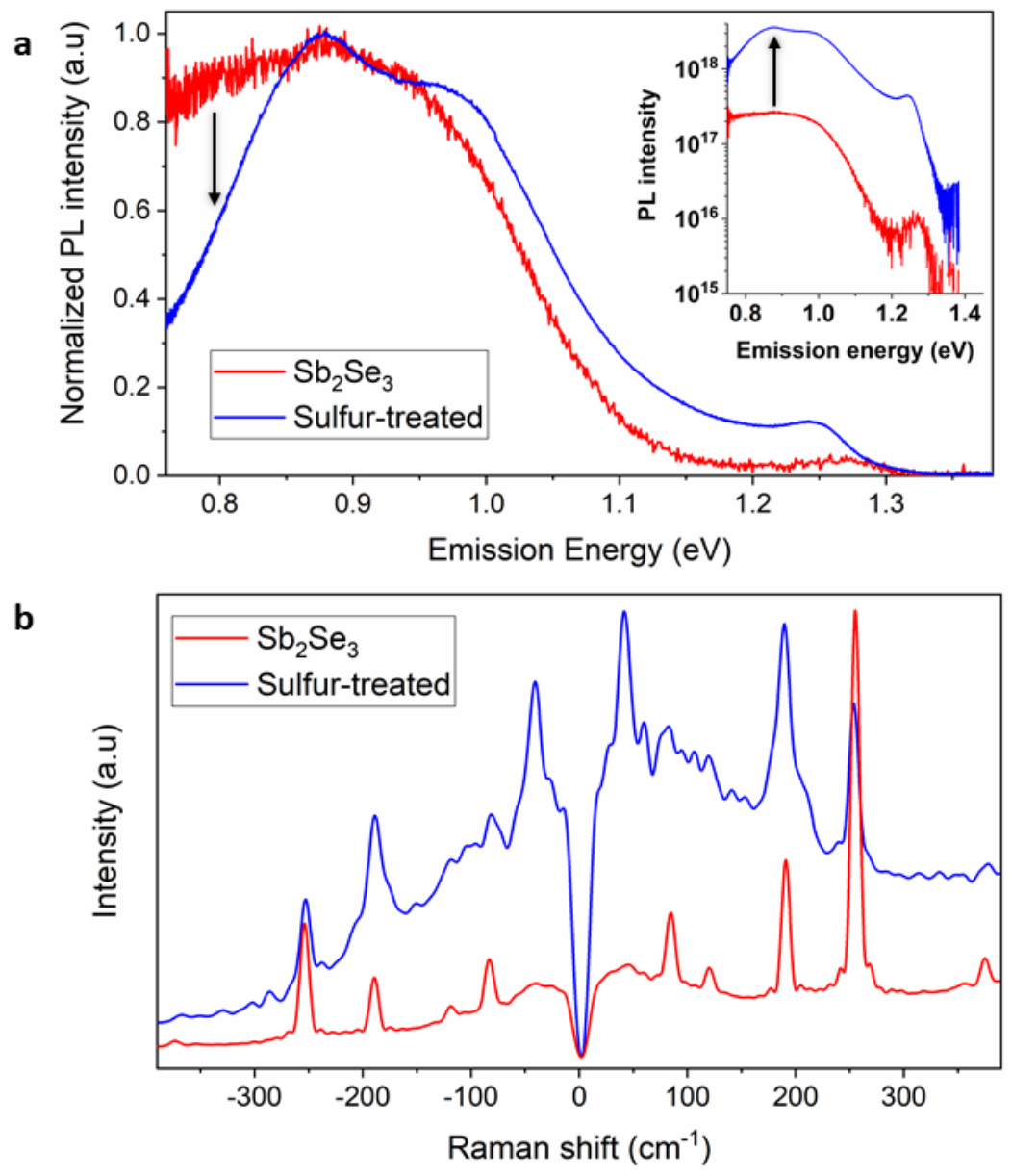

Figure 4. Defect passivation in sulfur-treated $\mathrm{Sb}_{2} \mathrm{Se}_{3}$ films. a Low temperature (10 K) normalized PL spectra of $\mathrm{Sb}_{2} \mathrm{Se}_{3}$ and sulfur-treated films (excitation wavelength $633 \mathrm{~nm}$ ). Inset shows the non-normalized PL intensity, illustrating the increased radiative recombination due to sulfur treatment. b LFRS spectra of $\mathrm{Sb}_{2} \mathrm{Se}_{3}$ films before and after defect passivation using sulfur.

Low-frequency Raman spectroscopy (LFRS) can be used to probe the acoustic vibrational modes (AVM) of materials. Long-range order induces AVM enhancement thus revealing otherwise hidden LFRS modes. ${ }^{[21,22]}$ AVMs of $\mathrm{Sb}_{2} \mathrm{Se}_{3}$ have been extensively studied using DFT-based theoretical models. Figure 4b represents the LFRS spectrum of the $\mathrm{Sb}_{2} \mathrm{Se}_{3}$ samples before and after sulfur treatment. Experimental LFRS studies have optically resolved 15 of these theoretically predicted modes. The theoretical and experimental data agree within 5\% deviation from theory. ${ }^{[23-}$ ${ }^{25]}$ The AVM modes reported in the literature (theoretically as well as experimentally) are compared with the modes we observed, in Table S1, and a graphical summary is presented in Figure S11. All of the LFRS peaks observed prior to sulfur treatment agree with the previously reported modes, the vibrational symmetries of which are well documented in the literature. ${ }^{[24,25]}$ Upon sulfur 
treatment, two new AVM peaks emerge in the LFRS at $95.18 \mathrm{~cm}^{-1}$ and $141.41 \mathrm{~cm}^{-1}$, corresponding to the simulated values of $94 \mathrm{~cm}^{-1}$ and $142 \mathrm{~cm}^{-1}$, respectively. We believe that this is the first time these modes have been resolved optically. We also observe that sulfur treatment enhances the AVMs at $106.53 \mathrm{~cm}^{-1}$ and $118.83 \mathrm{~cm}^{-1}$. Furthermore, the sulfur treatment induced a blue shift in the position of the LFRS peaks from $60.03 \mathrm{~cm}^{-1}, 84.81 \mathrm{~cm}^{-1}, 191.20 \mathrm{~cm}^{-1}$ and $256.03 \mathrm{~cm}^{-1}$, observed prior to sulfur treatment, to lower wavenumbers of $59.05 \mathrm{~cm}^{-1}, 82.83 \mathrm{~cm}^{-1}, 189.74 \mathrm{~cm}^{-1}$, and $253.07 \mathrm{~cm}^{-1}$, respectively. The LFRS peaks observed at $27.69 \mathrm{~cm}^{-1}, 42.65 \mathrm{~cm}^{-1}$ and $153.65 \mathrm{~cm}^{-1}$ match those of elemental sulfur. While the peak at $27.69 \mathrm{~cm}^{-1}$ can be attributed to the external surface vibrations of $\alpha-\mathrm{S}_{8}$ and the peak at $42.65 \mathrm{~cm}^{-1}$ can be attributed to similar surface modes of $\gamma-\mathrm{S}_{8}$, the peak at $153.65 \mathrm{~cm}^{-1}$ is attributed to internal bond vibration of $\mathrm{S}_{8}$ as consistent with reports in the literature that all the allotropes of $\mathrm{S}_{8}$ have similar spectral feature at $153 \mathrm{~cm}^{-1}{ }^{[26]}$ The changes we observe in the LFRS of $\mathrm{Sb}_{2} \mathrm{Se}_{3}$ can be attributed to defect passivation upon sulfur treatment. Based on these observations, we propose that defect passivation induces long range order in the system, which results in enhancement of hidden AVM modes of $\mathrm{Sb}_{2} \mathrm{Se}_{3}$. It can also be inferred that the presence of sulfur allotropes at defect states induces new local force-fields that drive the material to reorient. This reorientation results in long range order, enhancement of hidden AVMs and blue-shifts in the LFRS peaks.

The defect passivation in sulfur-treated $\mathrm{Sb}_{2} \mathrm{Se}_{3}$ was further confirmed by investigating the photovoltage generated from $\mathrm{Sb}_{2} \mathrm{Se}_{3}$ PEC devices. To confirm the improvement of photovoltage upon sulfurization treatment of $\mathrm{Sb}_{2} \mathrm{Se}_{3}$, dual working electrode (DWE) devices were fabricated to directly probe the photovoltage generated by a p-n junction in a water splitting photocathode (in our case $\mathrm{Sb}_{2} \mathrm{Se}_{3} / \mathrm{TiO}_{2}$ ). As shown in Figure S12, the sulfurized sample (sulfur-treated $/ \mathrm{TiO}_{2} / \mathrm{Pt}$ ) exhibits an improved onset potential of $\sim 350 \mathrm{mV}$ vs $\mathrm{V}_{\mathrm{RHE}}$, compared to the non-treated $\mathrm{Sb}_{2} \mathrm{Se}_{3} / \mathrm{TiO}_{2} / \mathrm{Pt}$ whose onset potential is $\sim 210 \mathrm{mV}$ vs $\mathrm{V}_{\mathrm{RHE}}$. The $\mathrm{J}-\Delta \mathrm{V}$ ( $\Delta \mathrm{V}$ is the difference between the back contact potential WE1 and surface potential WE2) curves indicate that the $\mathrm{V}_{\text {oc }}$ generated by the $\mathrm{Sb}_{2} \mathrm{Se}_{3} / \mathrm{TiO}_{2}$ heterojunction is $\sim 210 \mathrm{mV}$, which agrees well with the value of the onset potential for the standard J-V curve. Figure S12 clearly illustrates the improvement of photovoltage upon sulfurization treatment. Improvement in the photovoltage would indicate passivation of defects in the semiconductor and hence would be a key strategy for the improvement of the efficiency of $\mathrm{Sb}_{2} \mathrm{Se}_{3}$ for PEC and PV applications. ${ }^{[27]}$ 


\section{CONCLUSION}

A comprehensive investigation of the charge carrier dynamics of $\mathrm{Sb}_{2} \mathrm{Se}_{3}$ and the influence of sulfur treatment was performed using time resolved microwave conductivity (TRMC), photoluminescence (PL) spectroscopy and low frequency Raman spectroscopy (LFRS) to gain fundamental understanding of the recombination pathways in $\mathrm{Sb}_{2} \mathrm{Se}_{3}$. The key finding is that upon sulfur treatment the carrier lifetime is increased (i.e. reduction of electron-hole recombination) by the passivation of deep defects in $\mathrm{Sb}_{2} \mathrm{Se}_{3}$, which is evidenced by two key observations. First, the lifetime of the fast process (which corresponds to electron-hole recombination) is increased while the lifetime of the carrier trapping and de-trapping process (which corresponds to reduced defect states) is decreased for the sulfurized film. Moreover, it was shown that the sulfurization treatment increases the lifetime of electron-hole recombination across all wavelengths (even for long wavelengths close to the bandgap energy) indicating that the treatment improves carrier lifetime in both the surface and bulk regions. The passivation of deep defects in $\mathrm{Sb}_{2} \mathrm{Se}_{3}$ also increased radiative recombination-a key requisite for high efficiency PV and PEC devices. The improved charge carrier dynamics is also supported by LFRS which shows improved long-range order in the $\mathrm{Sb}_{2} \mathrm{Se}_{3}$ thin films upon sulfur treatment, as evidenced by the enhancement of hidden AVMs and blue-shifts in the LFRS peaks. Unlocking the defect passivation mechanisms in $\mathrm{Sb}_{2} \mathrm{Se}_{3}$ provides crucial insights on the electronic properties of this emerging PV/PEC material, paving the way for highly efficient photoconversion devices.

\section{EXPERIMENTAL SECTION}

Synthesis of $\mathrm{Sb}_{2} \mathrm{Se}_{3}$ thin films, sulfurization and material characterization: $\mathrm{Sb}$ metal was sputtered from a high purity Sb sputtering target to generate films of $300 \mathrm{~nm}$ thickness on quartz substrates for time resolved microwave conductivity (TRMC) measurements, and on Au-coated FTO substrates for dual working electrode measurements. Selenization and sulfurization procedures were the same as used in our previous work. ${ }^{[6]}$ The crystal structure of $\mathrm{Sb}_{2} \mathrm{Se}_{3}$ and sulfur-treated $\mathrm{Sb}_{2} \mathrm{Se}_{3}$ was determined by X-ray diffraction using a Rigaku SmartLab instrument ( $\mathrm{Cu} \mathrm{K} \mathrm{K}_{\alpha}$ radiation). UV-vis absorption spectra were measured using a PerkinElmer Lambda 950 spectrometer fitted with an integrating sphere. X-ray photoelectron spectroscopy (XPS) was conducted using a Physical Electronics (PHI) Quantum 2000 X-ray photoelectron spectrometer 
featuring monochromatic Al-K $\alpha$ radiation, generated from an electron beam operated at $15 \mathrm{kV}$ and 32.3 W. The energy scale of the instrument was calibrated using $\mathrm{Au}$ and $\mathrm{Cu}$ reference samples. The analysis was conducted at $1 \times 10^{-6} \mathrm{~Pa}$, with an electron take off angle of $45^{\circ}$ and a pass energy of $46.00 \mathrm{eV}$. Charge compensation during the measurement was achieved using a low energy electron source. The sputter depth profile was performed using Ar ions ( $2 \mathrm{kV}$ potential) on an area of approximately $4 \mathrm{~mm}^{2}$. Surface elemental concentrations were determined using the instrument specific sensitivity factors for calculation. The core level emissions were fitted to deconvolute spectra with contributions from multiple elements using Voigt profiles (GL30) after Shirley background subtraction.

Time-resolved microwave conductivity measurements: TRMC measurements were performed by mounting the samples in a microwave cavity cell and placing within a setup similar to the one described. ${ }^{[16,28]}$ A voltage controlled oscillator (SiversIMA VO3262X) generated the microwaves (X-band region, 8.4-8.7 GHz). During the measurements, a change in the microwave power $(\Delta P / P)$ reflected by the cavity upon sample excitation by 3 ns (full-width at half-maximum) pulses of a wavelength tunable optical parametric oscillator (OPO) coupled to a diode-pumped Q-switched $\mathrm{Nd}$ :YAG laser at wavelengths between 350 and $1100 \mathrm{~nm}$ (50 Hz repetition rate) was monitored and correlated to the photoinduced change in the conductance of the sample, $\Delta G$, given by

$$
\frac{\Delta P}{P}(t)=-K \Delta G(t)
$$

where $K$ is the sensitivity factor derived from the resonance characteristics of the cavity and the dielectric properties of the medium. From the experimentally observed change in the photoconductance, the product of the charge carrier generation yield $(\phi)$ and the sum of electron and hole mobilities $(\Sigma \mu)$ can be obtained

$$
\Phi \Sigma \mu=\frac{\Delta G_{\max }}{I_{0} \beta e F_{A}}
$$

where $I_{0}$ is the incident intensity per pulse, $\mathrm{e}$ is the elementary charge, $\beta$ is the ratio between the inner broad and narrow dimensions of the waveguide, $\mathrm{F}_{\mathrm{A}}$ is the fraction of incident photons absorbed within the sample, $\varphi$ is the charge carrier generation yield, and $\Sigma \mu$ is the sum of electron and hole mobilities Neutral density filters were selected to adjust the light intensity in the range of $10^{9}-10^{13}$ photons / pulse $/ \mathrm{cm}^{2}$. 
Photoluminescence spectroscopy: Low temperature photoluminescence measurements were carried out in a home-built system equipped with a helium flow cryostat under 663-nm wavelength diode laser excitation source. The emitted luminescence was collected through off-axis positioned parabolic mirrors and focused into a fiber, and spectrally resolved by a monochromator and subsequently detected by a Si-charge-coupled device (CCD) and an InGaAs diode array. All the data were corrected with a calibration lamp with a known spectrum. Neutral density filters were used to vary the power for excitation intensity dependent PL.

Low frequency Raman spectroscopy (LFRS): LFRS measurements were carried out using an integrated laser and Volume Holographic (VHG) filter system (ONDAX, XLF-MICRO $532 \mathrm{~nm}$ ) with $50 \mathrm{~mW}$ of optical power at an excitation wavelength of $\lambda_{\mathrm{ex}}=532 \mathrm{~nm}$. The laser output was routed into a lab microscope, and the Raman signal was fibre-coupled into an imaging spectrometer (Princeton Instruments, SP-2500i) with an EM-CCD camera (Princeton Instruments, ProEM 16002). Acquisition times of 5 seconds were used, and a grating groove density of $1800 \mathrm{~g} / \mathrm{mm}$ was selected. The measurements were carried out by first setting the focus to the top surface and then slightly lowering the focal plane into the depth of the sample to avoid edge effects. At least 3 different regions were measured for each sample. The spectral baselines were shifted for presentation purposes. The integration time for spectral collection was $5 \mathrm{~s}$ per acquisition. Each spectrum was recorded by accumulating 3 frames, yielding an overall integration time of $15 \mathrm{~s}$. The Raman frequencies were calibrated using a silicon wafer. All experiments were conducted at room temperature $(293 \mathrm{~K})$.

Dual-working electrode (DWE) fabrication: For DWE measurements, the $\mathrm{Sb}_{2} \mathrm{Se}_{3}$ was synthesized as described above. The main difference when compared to the conventional single working electrode setup is that a second working electrode (WE2, which measures the surface potential) is fabricated on the catalyst $(\mathrm{Pt})$, which enables the determination of the photovoltage (the difference between the back contact potential (WE1) and the front contact potential (WE2)). The exposed Au-coated FTO served as the WE1 or back contact. To make the WE2 (or front contact), a $20 \mathrm{~nm}$-thick Au layer was sputtered onto a part of the exposed Pt layer, as shown in Figure S12. A copper wire was then connected to the Au via Ag paint as the WE2 and covered with another layer of epoxy for protection from the electrolyte. 


\section{ACKNOWLEDGEMENTS}

S. David Tilley thanks the Swiss National Science Foundation (AP Energy Grant PYAPP2 160586) and the University Research Priority Program LightChEC for funding. Sudhanshu Shukla acknowledges funding from Luxembourgish Fond National de la Recherche FNR through the MASSENA project. Yaakov R. Tischler acknowledges grant number 205509 Israel ministry of science and technology (MOST) optoelectronics grant for funding.

\section{REFERENCES}

[1] W. Yang, J. Moon, J. Mater. Chem. A 2019, 7, 20467.

[2] A. Mavlonov, T. Razykov, F. Raziq, J. Gan, J. Chantana, Y. Kawano, T. Nishimura, H. Wei, A. Zakutayev, T. Minemoto, X. Zu, S. Li, L. Qiao, Sol. Energy 2020, 201, 227.

[3] Y. Zhou, L. Wang, S. Chen, S. Qin, X. Liu, J. Chen, D.-J. Xue, M. Luo, Y. Cao, Y. Cheng, E. H. Sargent, J. Tang, Nat Phot. 2015, 9, 409.

[4] C. N. Savory, D. O. Scanlon, J. Mater. Chem. A 2019, 7, 10739.

[5] Y. Ma, B. Tang, W. Lian, C. Wu, X. Wang, H. Ju, C. Zhu, F. Fan, T. Chen, J. Mater. Chem. A 2020, 8, 6510 .

[6] R. R. Prabhakar, W. Septina, S. Siol, T. Moehl, R. Wick-Joliat, S. D. Tilley, J. Mater. Chem. A 2017, 5, 23139.

[7] H. Zhou, M. Feng, M. Feng, X. Gong, D. Zhang, Y. Zhou, S. Chen, Appl. Phys. Lett. 2020, $116,113902$.

[8] C. Swiatkowski, A. Sanders, K. -D. Buhre, M. Kunst, J. Appl. Phys. 1995, 78, 1763.

[9] M. Kunst, F. Wünsch, D. Fuertes Marrón, A. Meeder, Thin Solid Films 2004, 451-452, 152.

[10] E. M. Hutter, G. E. Eperon, S. D. Stranks, T. J. Savenije, J. Phys. Chem. Lett. 2015, 6, 3082 .

[11] A. Kay, M. Fiegenbaum-Raz, S. Müller, R. Eichberger, H. Dotan, R. van de Krol, F. F. Abdi, A. Rothschild, D. Friedrich, D. A. Grave, Adv. Funct. Mater. 2019, 0, 1901590. 
[12] M. S. Prévot, X. A. Jeanbourquin, W. S. Bourée, F. Abdi, D. Friedrich, R. van de Krol, N. Guijarro, F. Le Formal, K. Sivula, Chem. Mater. 2017, 29, 4952.

[13] F. F. Abdi, T. J. Savenije, M. M. May, B. Dam, R. van de Krol, J. Phys. Chem. Lett. 2013, $4,2752$.

[14] M. Kölbach, I. J. Pereira, K. Harbauer, P. Plate, K. Höflich, S. P. Berglund, D. Friedrich, R. van de Krol, F. F. Abdi, Chem. Mater. 2018, 30, 8322.

[15] S. Feldmann, S. Macpherson, S. P. Senanayak, M. Abdi-Jalebi, J. P. H. Rivett, G. Nan, G. D. Tainter, T. A. S. Doherty, K. Frohna, E. Ringe, R. H. Friend, H. Sirringhaus, M. Saliba, D. Beljonne, S. D. Stranks, F. Deschler, Nat. Photonics 2020, 14, 123.

[16] T. J. Savenije, A. J. Ferguson, N. Kopidakis, G. Rumbles, J. Phys. Chem. C 2013, 117, 24085 .

[17] Y. Bi, E. M. Hutter, Y. Fang, Q. Dong, J. Huang, T. J. Savenije, J. Phys. Chem. Lett. 2016, $7,923$.

[18] T. Unold, L. Gütay, Adv. Charact. Tech. Thin Film Sol. Cells 2016, 275.

[19] C. Spindler, T. Galvani, L. Wirtz, G. Rey, S. Siebentritt, J. Appl. Phys. 2019, 126, 175703.

[20] A. Stoliaroff, A. Lecomte, O. Rubel, S. Jobic, X. Zhang, C. Latouche, X. Rocquefelte, ACS Appl. Energy Mater. 2020, 3, 2496.

[21] R. T. Sam, T. Umakoshi, P. Verma, Appl. Phys. Express 2020, 13, 072003.

[22] M. Inoue, H. Hisada, T. Koide, J. Carriere, R. Heyler, T. Fukami, Org. Process Res. Dev. 2017, 21, 262.

[23] A. Shongalova, M. R. Correia, B. Vermang, J. M. V. Cunha, P. M. P. Salomé, P. A. Fernandes, MRS Commun. 2018, 8, 865 .

[24] N. Fleck, T. D. C. Hobson, C. N. Savory, J. Buckeridge, T. D. Veal, M. R. Correia, D. O. Scanlon, K. Durose, F. Jäckel, J. Mater. Chem. A 2020, 8, 8337.

[25] J. Ibáñez, J. A. Sans, C. Popescu, J. López-Vidrier, J. J. Elvira-Betanzos, V. P. CuencaGotor, O. Gomis, F. J. Manjón, P. Rodríguez-Hernández, A. Muñoz, J. Phys. Chem. C 2016, 120, 10547. 
[26] C. Nims, B. Cron, M. Wetherington, J. Macalady, J. Cosmidis, Sci. Rep. 2019, 9, DOI 10.1038/s41598-019-44353-6.

[27] S. Siebentritt, Nat. Energy 2017, 2, 840.

[28] J. E. Kroeze, T. J. Savenije, J. M. Warman, J. Am. Chem. Soc. 2004, 126, 7608. 


\section{Table of Contents}

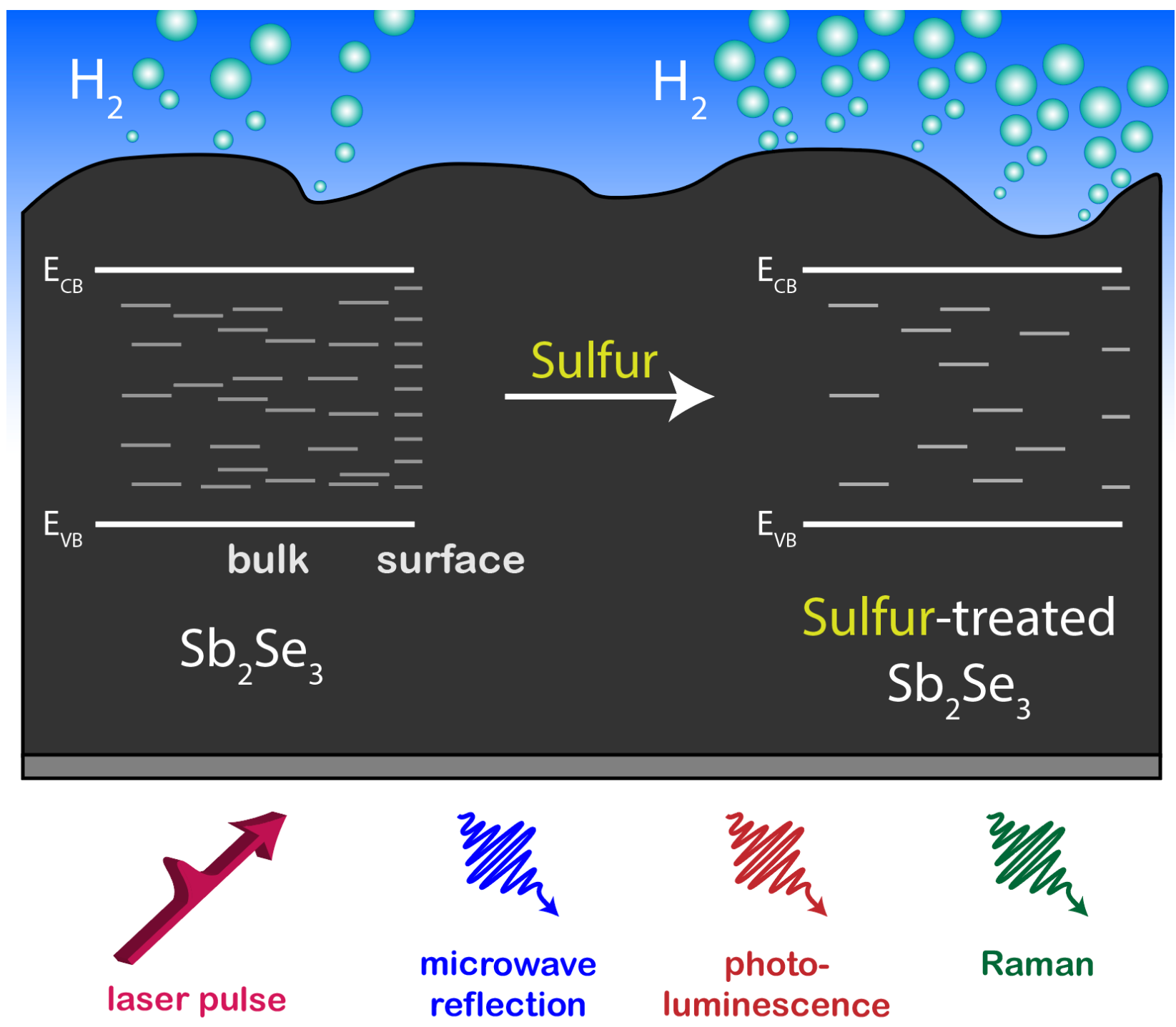

Time-resolved microwave conductivity reveals that a low temperature sulfurization treatment reduces both surface and bulk recombination in thin film $\mathrm{Sb}_{2} \mathrm{Se}_{3}$-based photocathodes for photoelectrochemical water splitting. Photoluminescence shows an improved radiative recombination yield, and low frequency Raman spectroscopy indicates improved long range order in the films following sulfur treatment. 